\title{
The formation, disruption and properties of pressure-supported stellar systems and implications for the astrophysics of galaxies
}

\author{
Pavel Kroupa \\ Argelander Institute for Astronomy, Auf dem Hügel 71, D 53121 Bonn, Germany \\ email: pavel@astro.uni-bonn.de
}

\begin{abstract}
Most stars form in dense star clusters deeply embedded in residual gas. These objects must therefore be seen as the fundamental building blocks of galaxies. With this contribution some physical processes that act in the very early and also later dynamical evolution of dense stellar systems in terms of shaping their later appearance and properties, and the impact they have on their host galaxies, are highlighted. Considering dense systems with increasing mass, it turns out that near $10^{6} M_{\odot}$ their properties change fundamentally: stellar populations become complex, a galaxial mass-radius relation emerges and the median two-body relaxation time becomes longer than a Hubble time. Intriguingly, only systems with a two-body relaxation time longer than a Hubble time show weak evidence for dark matter, whereby dSph galaxies form total outliers.
\end{abstract}

Keywords. stars: formation, stars: luminosity function, mass function, globular clusters: general, galaxies: formation

\section{Embedded clusters}

Fragmentation: The very early stages of cluster evolution on a scale of a few pc are dominated by gravitational fragmentation of a turbulent magnetized contracting molecular cloud core (Clarke, Bonnell \& Hillenbrand 2000; Tilley \& Pudritz 2007; Mac Low \& Klessen 2004). The existing simulations show the formation of contracting filaments which fragment into denser cloud cores that form sub-clusters of accreting proto-stars. As soon as the proto-stars emit radiation and outflows of sufficient energy and momentum to affect the cloud core these simulations become expensive as radiative transport and deposition of momentum and mechanical energy by non-isotropic outflows are difficult to handle with given present computational means (Stamatellos et al. 2007; Dale, Ercolano \& Clarke 2007).

Observations of the very early stages at times $\lesssim$ few $10^{5}$ yr suggest proto-clusters to have a hierarchical proto-stellar distribution: a number of sub-clusters with radii $\lesssim 0.2 \mathrm{pc}$ and separated in velocity space are often seen embedded within a region less than a pc across (Testi et al. 2000). Most of these sub-clusters may merge to form a more massive embedded cluster (Scally \& Clarke 2002; Fellhauer \& Kroupa 2005).

Mass segregation: Whether or not star clusters or sub-clusters form mass-segregated remains an open issue. Mass segregation by birth is a natural expectation because protostars near the density maximum of the cluster have more material to accrete. For these, the ambient gas is at a higher pressure allowing proto-stars to accrete longer before feedback termination stops further substantial gas inflow (Zinnecker \& Yorke 2007). Initially mass-segregated sub-clusters preserve mass segregation upon merging (McMillan, Versperini \& Portegies Zwart 2007). However, in dense proto-clusters (thousands of stars 
within less than a pc), the energy equipartition time-scale between the stars is very short such that mass segregation may formally occur dynamically within one to a few crossing times (Kroupa 2002a). Currently we cannot say conclusively if mass segregation is a birth phenomenon (e.g. Gouliermis et al. 2004), or whether the more massive stars form anywhere throughout the proto-cluster volume. Star clusters that have already blown out their gas are typically mass-segregated (e.g. R136, Orion Nebula Cluster).

Affirming natal mass segregation would impact positively on the notion that massive stars $\left(\gtrsim 10 M_{\odot}\right)$ only form in rich clusters, and negatively on the suggestion that they can also form in isolation (for recent work on this topic see Li, Klessen \& Mac Low (2003); Parker \& Goodwin (2007)).

Feedback termination: The star-formation efficiency (SFE), $\epsilon \equiv M_{\mathrm{ecl}} /\left(M_{\mathrm{ecl}}+\right.$ $\left.M_{\text {gas }}\right)$, where $M_{\text {ecl }}, M_{\text {gas }}$ are the mass in freshly formed stars and residual gas, respectively, is $0.2 \lesssim \epsilon \lesssim 0.4$ (Lada \& Lada 2003) implying that the physics dominating the star-formation process on scales less than a few pc is stellar feedback. Within this volume, the pre-cluster cloud core contracts under self gravity thereby forming stars ever more vigorously, until feedback energy suffices to halt the process (feedback-termination).

Dynamical state at feedback termination: Each proto-star needs about $t_{\mathrm{ps}} \approx$ $10^{5} \mathrm{yr}$ to accumulate about $95 \%$ of its mass (Wuchterl \& Tscharnuter 2003). The protostars form throughout the pre-cluster volume as the proto-cluster cloud core contracts. The overall pre-cluster cloud-core contraction until feedback-termination takes $t_{\mathrm{cl} \text {,form }} \approx$ few $\times(2 / \sqrt{G})\left(M_{\text {ecl }} / \epsilon\right)^{-\frac{1}{2}} R^{\frac{3}{2}}$ (a few times the crossing time), which is about the time over which the cluster forms. Once a proto-star condenses out of the hydro-dynamical flow it becomes a ballistic particle moving in the time-evolving cluster potential. Because many generations of proto-stars can form over the cluster-formation time-scale and if the crossing time through the cluster is a few times shorter than $t_{\mathrm{cl}, \text { form }}$, then the assumption may be made that the very young cluster is mostly virialised when star formation stops and before the removal of the residual gas. It is noteworthy that $t_{\mathrm{ps}} \approx t_{\mathrm{cl} \text {,form }}$ for $M_{\mathrm{ecl}} / \epsilon \approx$ $10^{4.5} M_{\odot}$ (proto-star formation time is comparable to the cluster formation time) which is near the turnover mass in the old-star-cluster mass function.

A critical parameter is thus the ratio $\tau=t_{\mathrm{cl}, \text { form }} / t_{\text {cross }}$. If it is less than unity then proto-stars "freeze out" of the gas and cannot virialise in the potential before the residual gas is removed. Such embedded clusters may be kinematically cold if the pre-cluster cloud core was contracting, or hot if the pre-cluster cloud core was pressure confined, because the young stars do not feel the gas pressure.

In those cases where $\tau>1$ the embedded cluster is approximately in virial equilibrium and the pre-gas-expulsion stellar velocity dispersion in the embedded cluster, $\sigma \approx \sqrt{G M_{\mathrm{ecl}} /(\epsilon R)}$, may reach $\sigma=40 \mathrm{pc} / \mathrm{Myr}$ if $M_{\mathrm{ecl}}=10^{5.5} M_{\odot}$ which is the case for $\epsilon R<1 \mathrm{pc}$. This is easily achieved since the radius of one-Myr old clusters is $R \approx 0.8$ pc with no dependence on mass. Some observationally explored cases are discussed by Kroupa (2005). Notably, using K-band number counts, Gutermuth et al. (2005) appear to find evidence for expansion after gas removal. Interestingly, recent Spitzer results suggest a scaling of $R$ with mass, $M_{\text {ecl }} \propto R^{2}$ (Allen et al. 2007), so the question how compact embedded clusters form and whether there is a mass-radius relation needs further clarification. I note that such a scaling is obtained for a stellar population that expands freely with a velocity given by the velocity dispersion in the embedded cluster. Is the observed scaling then a result of expansion from a compact birth configuration after gas expulsion?

There are two broad camps suggesting on the one hand side that molecular clouds and star clusters form on a free-fall time-scale (Elmegreen 2000; Hartmann 2003) and on the 
other that many free-fall times are needed (Krumholz \& Tan 2007). The former implies $\tau \approx 1$ while the latter implies $\tau>1$.

Thus, currently unclear issues concerning the initialisation of $N$-body models of embedded clusters is the ratio $\tau=t_{\mathrm{cl} \text {,form }} / t_{\mathrm{cross}}$, and whether a mass-radius relation exists for embedded clusters before the development of HII regions. To make progress I assume for now that the embedded clusters are in virial equilibrium at feedback termination $(\tau>1)$ and that they form highly concentrated with $R \lesssim 1 \mathrm{pc}$ independently of mass.

The mass of the most massive star: Young clusters show a well-defined correlation between the mass of the most massive star, $m_{\max }$, in dependence of the stellar mass of the embedded cluster, $M_{\text {ecl }}$, which appears to saturate at $m_{\max } \approx 150 M_{\odot}$ (Weidner \& Kroupa 2006). This may indicate feedback termination of star formation within the proto-cluster volume coupled to the most massive stars forming latest, or "turning-on" at the final stage of cluster formation (Elmegreen 1983). The physical maximum stellar mass near $150 M_{\odot}$ (Weidner \& Kroupa 2004; Figer 2005; Oey \& Clarke 2005; Koen 2006; Zinnecker \& Yorke 2007) must be a result of stellar structure stability, but may be near $80 M_{\odot}$ as predicted by theory if the most massive stars reside in near-equal componentmass binary systems (Kroupa \& Weidner 2005). It may also be that the calculated stellar masses are significantly overestimated (Martins, Schaerer \& Hillier 2005).

The cluster core of massive stars: Irrespectively of whether the massive stars ( $10 M_{\odot}$ ) form at the cluster centre or whether they segregate there due to energy equipartition, they ultimately form a compact sub-population that is dynamically highly unstable. Massive stars are ejected from such cores very efficiently on a core-crossing time-scale, and for example the well-studied Orion Nebula cluster (ONC) has probably already shot out 70 per cent of its stars more massive than $5 M_{\odot}$ (Pflamm-Altenburg \& Kroupa 2006). The properties of $\mathrm{O}$ and $\mathrm{B}$ runaway stars have been used by Clarke \& Pringle (1992) to deduce the typical birth configuration of massive stars, finding them to form in binaries with similar-mass components in compact small- $N$ groups devoid of low-mass stars. Among others, the core of the ONC is just such a system.

The star-formation history in a cluster: The detailed star-formation history in a cluster contains information about the events that build-up the cluster. Intriguing is the recent evidence for some clusters that while the bulk of the stars have ages different by less than a few $10^{5} \mathrm{yr}$, a small fraction of older stars are often harboured (Palla \& Stahler 2000). This may be interpreted to mean that clusters form over about 10 Myr with a final highly accelerated phase, in support of the notion that turbulence of a magnetized gas determine the early cloud-contraction phase (Krumholz \& Tan 2007).

A different interpretation would be that as a pre-cluster cloud core contracts on a free-fall time-scale, it traps surrounding field stars which thereby become formal cluster members: Most clusters form in regions of a galaxy that has seen previous star formation. The velocity dispersion of the previous stellar generation, such as an expanding OB association, is usually rather low, around a few $\mathrm{km} / \mathrm{s}$ to $10 \mathrm{~km} / \mathrm{s}$. The deepening potential of a newly-contracting pre-cluster cloud core will be able to capture some of the preceding generation of stars such that these older stars become formal cluster members although they did not form in this cluster. Pflamm-Altenburg \& Kroupa (2007) study this problem for the ONC showing that the reported age spread by Palla et al. (2007) can be accounted for in this way. This suggests that the star-formation history of the ONC may in fact not have started about $10 \mathrm{Myr}$ ago, supporting the argument by Elmegreen (2000) and Hartmann (2003) that clusters form on a timescale comparable to the crossing time of the pre-cluster cloud core.

For very massive clusters such as $\omega$ Cen, Fellhauer et al. (2006) show that the potential is sufficiently deep such that the pre-cluster cloud core may capture the field stars of a 
previously existing dwarf galaxy. Up to $30 \%$ or more of the stars in $\omega$ Cen may be captured field stars. This would be able to explain an age spread of a few Gyr in the cluster, and is consistent with the notion that $\omega$ Cen formed in a dwarf galaxy that was captured by the Milky Way. The attractive aspect of this scenario is that $\omega$ Cen need not have been located at the center of the incoming dwarf galaxy as a nucleus, but within its disk, because it opens a larger range of allowed orbital parameters for the putative dwarf galaxy moving about the Milky Way. The currently preferred scenario in which $\omega$ Cen was the nucleus of the dwarf galaxy implies that the galaxy was completely stripped while falling into the Milky Way leaving only its nucleus on its current retrograde orbit. The new scenario allows the dwarf galaxy to be absorbed into the Bulge of the MW with $\omega$ Cen being stripped from it on its way in.

Expulsion of residual gas: When the most massive stars are $\mathrm{O}$ stars they destroy the proto-cluster nebula and quench further star formation by first ionising most of it. The ionised gas, being now at a temperature near $10^{4} \mathrm{~K}$ and in serious over-pressure, pushes out and escapes the confines of the cluster volume with the sound speed (near $10 \mathrm{~km} / \mathrm{s}$ ) or faster if the winds being blown off $\mathrm{O}$ stars with velocities of thousands of $\mathrm{km} / \mathrm{s}$ impart sufficient momentum. In reality, this evolution is highly dynamic and can be described as an explosion (the cluster "pops"), and probably occurs non-spherically because the gas seeks low-density channels in the nebula which then allow the hot gas to escape (Dale et al. 2005).

If the clusters are more massive than about $10^{5} M_{\odot}$ such that the velocity dispersion is larger than the sound speed of the ionised gas, then the cluster reacts adiabatically because the stars move in a potential that varies more slowly than the stellar crossing time through the cluster. For clusters without $\mathrm{O}$ and massive B stars, nebula disruption probably occurs on the cluster-formation time-scale, $\approx 10^{6} \mathrm{yr}$, and the evolution is again adiabatic.

Kroupa \& Boily (2002) referred to clusters without O stars (stellar mass of the embedded cluster $M_{\mathrm{ecl}} \lesssim 10^{2.5} M_{\odot}$ ) as clusters of type I, those with O stars but with $10^{2.5} \lesssim M_{\mathrm{ecl}} / M_{\odot} \lesssim 10^{5.5}$ as type II clusters, and the very massive clusters $\left(M_{\mathrm{ecl}} \gtrsim\right.$ $10^{5.5} M_{\odot}$ ) as type III clusters. A type IV "cluster" may be added for extremely massive "clusters" for which only many supernovae are able to provide sufficient energy to blow out the residual gas $\left(M_{\mathrm{ecl}} \gtrsim 10^{7} M_{\odot}\right)$. This broad categorisation has easy-to-understand implications for the star-cluster mass function.

If clusters pop and which fraction of stars remain in a post-gas expulsion cluster depend critically on the ratio between the gas-removal time scale and the cluster crossing time. This ratio thus defines which clusters succumb to infant mortality, and which clusters suffer cluster infant weight loss. The well-studied cases do indicate that the removal of most of the residual gas does occur within a cluster-dynamical time, $\tau_{\text {gas }} / t_{\text {cross }} \lesssim 1$. Examples noted (Kroupa 2005) are the ONC and R136 in the LMC both having significant super-virial velocity dispersions. Other examples are the Treasure-Chest cluster and the very young star-bursting clusters in the massively-interacting Antennae galaxy which appear to have HII regions expanding at velocities such that the cluster volume may be evacuated within a cluster dynamical time.

A simple calculation of the amount of energy deposited by an $\mathrm{O}$ star into its surrounding cluster-nebula also suggests it to be larger than the nebula binding energy (Kroupa 2005). This, however, only gives at best a rough estimate of the rapidity with which gas can be expelled; an inhomogeneous distribution of gas leads to the gas removal occurring preferably along channels and asymmetrically, such that the overall gas-excavation process is highly non uniform and variable (Dale et al. 2005). Bastian \& Goodwin (2006) note that many young clusters have a radial-density profile signature expected if they are 
expanding rapidly, supporting the notion of fast gas blow out. Goodwin \& Bastian (2006) and de Grijs \& Parmentier (2007) also find the dynamical mass-to-light ratio of young clusters to be too large strongly implying they are in the process of expanding after gas expulsion. A more detailed $N$-body study by Baumgardt, Kroupa \& Parmentier (2008) considers the change of $\tau_{\text {gas }} / t_{\text {cross }}$ with the number of OB stars in clusters of varying mass by comparing the feedback energy in radiation and winds with the binding energy of the embedded cluster. These calculations support the sub-division of clusters into IV types suggested above.

Weidner et al. (2007) attempted to measure infant weight loss by using a sample of young but exposed Galactic clusters and applying the maximal-star-mass vs cluster mass relation from above to estimate the birth mass of these clusters. The uncertainties are large, but the data firmly suggest that the typical cluster looses at least about 50 per cent of its stars.

Mass loss from evolving stars: An old globular cluster with a turn-off mass near $0.8 M_{\odot}$ will have lost 30 per cent of the mass that remained in it after gas expulsion due to stellar evolution (Baumgardt \& Makino 2003). As the mass loss is most rapid during the earliest times after re-virialisation after gas expulsion, the cluster expands further during this time. This is nicely seen in the Lagrange radii of realistic cluster-formation models (Kroupa, Aarseth \& Hurley 2001).

\section{Some implications for the astrophysics of galaxies}

In general, the above have a multitude of implications for galactic and stellar astrophysics:

(a) The heaviest-star - star-cluster-mass correlation constrains feedback models of star cluster formation (Elmegreen 1983). It also implies that by adding up all IMFs in all young clusters in a galaxy, the integrated galaxial initial mass function (IGIMF) is steeper than the invariant stellar IMF observed in star clusters with important implications for the mass-metallicity relation of galaxies (Kroupa \& Weidner 2005)

(b) The deduction that type II clusters probably "pop" implies that young clusters will appear to an observer to be super-virial, i.e. to have a dynamical mass larger than the luminous mass (Bastian \& Goodwin 2006; de Grijs \& Parmentier 2007).

(c) It also implies that galactic fields can be heated, and may also lead to galactic thick-disks and stellar halos around dwarf galaxies (Kroupa 2002b).

(d) The variation of the gas expulsion time-scale among clusters of different type implies that the star-cluster mass function (CMF) is re-shaped rapidly, on a time-scale of a few ten Myr (Kroupa \& Boily 2002).

(e) Associated with this re-shaping of the cluster CMF is the natural production of population II stellar halos during cosmologically early star-formation bursts (Kroupa \& Boily 2002; Parmentier \& Gilmore 2007; Baumgardt, Kroupa \& Parmentier 2008).

Points $b-e$ are considered in more detail in what follows.

\subsection{Stellar associations, open clusters and moving groups}

As one of the important implications of point $b$, a cluster in the age range $1-50 \mathrm{Myr}$ will have an unphysical $M / L$ ratio because it is out of dynamical equilibrium rather than having an abnormal stellar IMF (Bastian \& Goodwin 2006; de Grijs \& Parmentier 2007).

Another implication is that a Pleiades-like open cluster would have been born in a very dense ONC-type configuration and that, as it evolves, a "moving-group-I" is established during the first few dozen Myr which comprises roughly $2 / 3 \mathrm{rd}$ of the initial stellar population and is expanding outwards with a velocity dispersion which is a function of the 
pre-gas-expulsion configuration (Kroupa, Aarseth \& Hurley 2001). These computations were in fact the first to demonstrate, using high-precision $N$-body modelling, that the re-distribution of energy within the cluster during the embedded phase and during the expansion phase leads to the formation of a substantial remnant cluster despite the inclusion of all physical effects that are disadvantageous for this to happen (explosive gas expulsion, low SFE $\epsilon=0.33$, Galactic tidal field and mass loss from stellar evolution and an initial binary-star fraction of 100 per cent). Thus, expanding OB associations may be related to star-cluster birth, and many OB associations ought to have remnant star clusters as nuclei (see also Clarke et al. (2005)).

As the cluster expands becoming part of an OB association, the radiation from its massive stars produce expanding HII regions that may trigger star formation Gouliermis et al. (e.g. 2007).

A "moving-group-II" establishes later as the "classical" moving group made-up of stars which slowly diffuse/evaporate out of the re-virialised cluster remnant with relative kinetic energy close to zero. The velocity dispersion of moving group I is thus comparable to the pre-gas-expulsion velocity dispersion of the cluster, while moving group II has a velocity dispersion close to zero.

\subsection{The velocity dispersion of galactic-field populations and galactic thick disks}

Thus, the moving-group-I would be populated by stars that carry the initial kinematical state of the birth configuration into the field of a galaxy. Each generation of star clusters would, according to this picture, produce overlapping moving-groups-I (and II), and the overall velocity dispersion of the new field population can be estimated by adding in quadrature all expanding populations. This involves an integral over the embeddedcluster mass function, $\xi_{\mathrm{ecl}}\left(M_{\mathrm{ecl}}\right)$, which describes the distribution of the stellar mass content of clusters when they are born. Because the embedded cluster mass function is known to be a power-law, this integral can be calculated for a first estimate (Kroupa $2002 \mathrm{~b}, 2005)$. The result is that for reasonable upper cluster mass limits in the integral, $M_{\text {ecl }} \lesssim 10^{5} M_{\odot}$, the observed age-velocity dispersion relation of Galactic field stars can be re-produced.

This theory can thus explain the much debated "energy deficit": namely that the observed kinematical heating of field stars with age could not, until now, be explained by the diffusion of orbits in the Galactic disk as a result of scattering on molecular clouds, spiral arms and the bar (Jenkins 1992). Because the velocity-dispersion for Galactic-field stars increases with stellar age, this notion can also be used to map the star-formation history of the Milky-Way disk by resorting to the observed correlation between the starformation rate in a galaxy and the maximum star-cluster mass born in the population of young clusters (Weidner, Kroupa \& Larsen 2004).

An interesting possibility emerges concerning the origin of thick disks. If the star formation rate was sufficiently high about $11 \mathrm{Gyr}$ ago, then star clusters in the disk with masses up to $10^{5.5} M_{\odot}$ would have been born. If they popped a thick disk with a velocity dispersion near $40 \mathrm{~km} / \mathrm{s}$ would result naturally (Kroupa 2002b). The notion for the origin of thick disks appears to be qualitatively supported by the observations of Elmegreen, Elmegreen \& Sheets (2004) who find galactic disks at a redshift between 0.5 and 2 to show massive star-forming clumps.

\subsection{Structuring the initial cluster mass function}

Another potentially important implication from this theory of the evolution of young clusters is that if the gas-expulsion-time-to-crossing-time ratio and/or the SFE varies with initial (embedded) cluster mass, then an initially featureless power-law mass function of 
embedded clusters will rapidly evolve to one with peaks, dips and turnovers at cluster masses that characterize changes in the broad physics involved.

As an example, Kroupa \& Boily (2002) assumed that the function $M_{\text {icl }}=f_{\text {st }} M_{\text {ecl }}$ exists, where $M_{\text {ecl }}$ is as above, $M_{\text {icl }}$ is the "classical initial cluster mass" and $f_{\text {st }}=f_{\text {st }}\left(M_{\text {ecl }}\right)$. The "classical initial cluster mass" is that mass which is inferred by classical $N$-body computations without gas expulsion (i.e. in effect assuming $\epsilon=1$, which is however, unphysical). Thus, for example, for the Pleiades, $M_{\mathrm{cl}} \approx 1000 M_{\odot}$ at the present time (age about $100 \mathrm{Myr}$ ). A classical initial model would place the initial cluster mass near $M_{\text {icl }} \approx 1500 M_{\odot}$ by using standard $N$-body calculations to quantify the secular evaporation of stars from an initially bound and virialised "classical" cluster (Portegies Zwart et al. 2001). If, however, the SFE was 33 per cent and the gas-expulsion time-scale was comparable to or shorter than the cluster dynamical time, then the Pleiades would have been born in a compact configuration resembling the ONC and with a mass of embedded stars of $M_{\mathrm{ecl}} \approx 4000 M_{\odot}$ (Kroupa, Aarseth \& Hurley 2001). Thus, $f_{\text {st }}\left(4000 M_{\odot}\right)=0.38(=1500 / 4000)$.

By postulating that there exist three basic types of embedded clusters, namely clusters without $\mathrm{O}$ stars (type I: $M_{\mathrm{ecl}} \lesssim 10^{2.5} M_{\odot}$, e.g. Taurus-Auriga pre-main sequence stellar groups, $\rho \mathrm{Oph}$ ), clusters with a few $\mathrm{O}$ stars (type II: $10^{2.5} \lesssim M_{\mathrm{ecl}} / M_{\odot} \lesssim 10^{5.5}$, e.g. the $\mathrm{ONC}$ ) and clusters with many $\mathrm{O}$ stars and with a velocity dispersion comparable to or higher than the sound velocity of ionized gas (type III: $M_{\mathrm{ecl}} \gtrsim 10^{5.5} M_{\odot}$ ) it can be argued that $f_{\mathrm{st}} \approx 0.5$ for type I, $f_{\mathrm{st}}<0.5$ for type II and $f_{\mathrm{st}} \approx 0.5$ for type III. The reason for the high $f_{\text {st }}$ values for types I and III is that gas expulsion from these clusters may be longer than the cluster dynamical time because there is no sufficient ionizing radiation for type I clusters, or the potential well is too deep for the ionized gas to leave (type III clusters). Type II clusters undergo a disruptive evolution and witness a high "infant mortality rate" (Lada \& Lada 2003), therewith being the pre-cursors of OB associations and Galactic clusters.

Under these conditions and an assumed functional form for $f_{\mathrm{st}}=f_{\mathrm{st}}\left(M_{\mathrm{ecl}}\right)$, the powerlaw embedded cluster mass function transforms into a cluster mass function with a turnover near $10^{5} M_{\odot}$ and a sharp peak near $10^{3} M_{\odot}$ (Kroupa \& Boily 2002). This form is strongly reminiscent of the initial globular cluster mass function which is inferred by e.g. Vesperini (1998, 2001); Parmentier \& Gilmore (2005); Baumgardt (1998) to be required for a match with the evolved cluster mass function that is seen to have a universal turnover near $10^{5} M_{\odot}$.

This analytical formulation of the problem has been verified nicely using $N$-body simulations combined with a realistic treatment of residual gas expulsion by Baumgardt, Kroupa \& Parmentier (2008), who show the Milky-Way globular cluster mass function to emerge from a power-law embedded-cluster mass function. Parmentier et al. (2008) expand on this by studying the effect that different assumptions on the physics of gas removal have on shaping the star-cluster mass function within about $50 \mathrm{Myr}$.

The general ansatz that residual gas expulsion plays a dominant role in early cluster evolution may thus bear the solution to the long-standing problem that the deduced initial cluster mass function needs to have this turnover, while the observed mass functions of young clusters are feature-less power-law distributions.

\subsection{The origin of population II stellar halos}

The above theory implies naturally that a major field-star component is generated whenever a population of star clusters forms. About 11 Gyr ago, the MW began its assembly by an initial burst of star formation throughout a volume spanning about $10 \mathrm{kpc}$ in radius. In this volume, the star formation rate must have reached $10 M_{\odot} / \mathrm{yr}$ such that star 
clusters with masses up to $\approx 10^{6} M_{\odot}$ formed (Weidner, Kroupa \& Larsen 2004), probably in a chaotic, turbulent early interstellar medium. The vast majority of embedded clusters suffered infant weight loss or mortality, the surviving long-lived clusters evolving to globular clusters. The so generated field population is the spheroidal population II halo, which has the same chemical properties as the surviving (globular) star clusters, apart from enrichment effects evident in the most massive clusters. All of these characteristics emerge naturally in the above model, as pointed out by Kroupa \& Boily (2002), by Parmentier \& Gilmore (2007) and most recently by Baumgardt, Kroupa \& Parmentier (2008).

\section{Long term, or classical, cluster evolution}

The long-term evolution of star clusters that survive infant weight loss and the mass loss from evolving stars is characterised by three physical processes: the drive of the self-gravitating system towards energy equipartition, stellar evolution processes and the heating or forcing of the system through external tides. One emphasis of star-cluster work in this context is on testing stellar-evolution theory and on the interrelation of stellar astrophysics with stellar dynamics given that the stellar-evolution and the dynamicalevolution time-scales are comparable. The reader is directed to other chapters in this book for further details.

Tidal tails: Tidal tails contain the stars evaporating from long-lived star clusters (the moving group II above). Given that energy equipartition leads to a filtering in energy space of the stars that escape at a particular time, one expects a gradient in the stellar mass function progressing along a tidal tail towards the cluster such that the mass function becomes flatter, i.e. richer in more massive stars. This effect is difficult to detect, but for example the long tidal tails found emanating from Pal 5 (Odenkirchen et al. 2003) may show evidence for this. As emphasised by Odenkirchen et al. (2003), tidal tails have another very interesting use: they probe the gravitational potential of the Milky Way if the differential motions along the tidal tail can be measured. They are thus important future tests of gravitational physics.

Death: Nothing lasts forever, and star clusters that survive initial re-virialisation after residual gas expulsion and mass loss from stellar evolution ultimately "die" after evaporating all member stars leaving a binary or a long-lived highly hierarchical multiple system composed of near-equal mass components (de La Fuente Marcos 1997, 1998). Note that these need not be stars. These cluster remnants are interesting, because they may account for all hierarchical multiple stellar systems in the Galactic field (Goodwin \& Kroupa 2005) with the implication that they are not a product of star formation, but rather of star-cluster dynamics.

\section{What is a galaxy?}

Old star clusters, dwarf-spheroidal (dSph) and dwarf-elliptical (dE) galaxies as well as galactic bulges and giant elliptical (E) galaxies are all stellar-dynamical systems that are supported by random stellar motions, i.e. they are pressure-supported. But why is one class of these pressure supported systems referred to as "star clusters", while the others are "galaxies"? Is there some fundamental physical difference between these two classes of systems?

Considering the radius as a function of mass, it becomes apparent that systems with $M \lesssim 10^{6} M_{\odot}$ do not show a mass-radius relation (MRR) and have $R \approx 4$ pc. More massive objects, however, show a well-defined MRR. In fact, Dabringhausen, Hilker \& Kroupa 
(2008) find that the "massive compact objects" (MCOs), which have $10^{6} \lesssim M / M_{\odot} \lesssim 10^{8}$, lie on the MRR of giant E galaxies $\left(\approx 10^{13} M_{\odot}\right)$ down to normal E galaxies $\left(10^{11} M_{\odot}\right)$ : $R / \mathrm{pc}=10^{-3.15}\left(M / M_{\odot}\right)^{0.60 \pm 0.02}$. Noteworthy is that systems with $M \gtrsim 10^{6} M_{\odot}$ also sport complex stellar populations, while less massive systems have single-age, singlemetallicity populations. The median two-body relaxation time is longer than a Hubble time for $M \gtrsim 3 \times 10^{6} M_{\odot}$, and only for these systems is there evidence for a slight increase in the dynamical mass-to-light ratio. Intriguingly, $(M / L)_{V} \approx 2$ for $M<10^{6} M_{\odot}$, while $(M / L)_{V} \approx 5$ for $M>10^{6} M_{\odot}$ with a possible decrease for $M>10^{8} M_{\odot}$. Finally, the average stellar density maximises at $M=10^{6} M_{\odot}$ with about $3 \times 10^{3} M_{\odot} / \mathrm{pc}^{3}$.

Thus,

- the mass $10^{6} M_{\odot}$ appears to be special,

- stellar populations become complex above this mass,

- evidence for dark matter only appears in systems that have a median two-body relaxation time longer than a Hubble time,

- dSph galaxies are the only stellar-dynamical systems with $10<(M / L)_{V}<1000$ and as such are total outliers.

$M \approx 10^{6} M_{\odot}$ therefore appears to be a characteristic mass scale such that less-massive objects show characteristics of star clusters being well-described by Newtonian dynamics, while more massive objects show behaviour more typical of galaxies. Defining a galaxy as a stellar-dynamical object which has a median two-body relaxation time longer than a Hubble time, i.e. essentially a system with a smooth potential, may be an objective and useful way to define a "galaxy" (Kroupa 1998).

Why only smooth systems show evidence for dark matter remains at best a striking coincidence, at worst it may be symptomatic of a problem in understanding dynamics in such systems.

\section{References}

Allen, L., et al. 2007, Protostars and Planets V, 361

Baumgardt, H. 1998, A\& $\mathcal{E}$, 330, 480

Baumgardt, H. \& Makino, J. 2003, MNRAS, 340, 227

Baumgardt, H., Kroupa, P., \& Parmentier, P. 2008, MNRAS, submitted

Bastian, N. \& Goodwin, S. P. 2006, MNRAS, 369, L9

Clark, P. C., Bonnell, I. A., Zinnecker, H., \& Bate, M. R. 2005, MNRAS, 359, 809

Clarke, C. J., Bonnell, I. A., \& Hillenbrand, L. A. 2000, Protostars and Planets IV, 151

Clarke, C. J. \& Pringle, J. E. 1992, MNRAS, 255, 423

Dabringhausen, J., Hilker, M., \& Kroupa, P. 2008, MNRAS, submitted

Dale, J. E., Bonnell, I. A., Clarke, C. J., \& Bate, M. R. 2005, MNRAS, 358, 291

Dale, J. E., Ercolano, B., \& Clarke, C. J. 2007, MNRAS, 1056

de Grijs, R. \& Parmentier, G. 2007, Chinese Journal of Astronomy and Astrophysics, 7, 155

de La Fuente Marcos, R. 1997, A\&\&A, 322, 764

de La Fuente Marcos, R. 1998, A\&A, 333, L27

Elmegreen, B. G. 1983, MNRAS, 203, 1011

Elmegreen, B. G. 2000, ApJ, 530, 277

Elmegreen, D. M., Elmegreen, B. G., \& Sheets, C. M. 2004, ApJ, 603, 74

Fellhauer, M. \& Kroupa, P. 2005, ApJ, 630, 879

Fellhauer, M., Kroupa, P. \& Evans, N. W. 2006, MNRAS, 372, 338

Figer, D. F. 2005, Nature, 434, 192

Goodwin, S. P. \& Bastian, N. 2006, MNRAS, 373, 752

Goodwin, S. P. \& Kroupa, P. 2005, A\& A, 439, 565

Gouliermis, D., Keller, S. C., Kontizas, M., Kontizas, E., \& Bellas-Velidis, I. 2004, A\& A, 416, 137 
Gouliermis, D. A., Quanz, S. P., \& Henning, T. 2007, ApJ, 665, 306

Gutermuth, R. A., Megeath, S. T., Pipher, J. L., Williams, J. P., Allen, L. E., Myers, P. C., \& Raines, S. N. 2005, ApJ, 632, 397

Hartmann, L. 2003, ApJ, 585, 398

Jenkins, A. 1992, MNRAS, 257, 620

Koen, C. 2006, MNRAS, 365, 590

Kroupa, P. 1998, MNRAS, 300, 200

Kroupa, P. 2002, Science, 295, 82

Kroupa, P. 2002, MNRAS, 330, 707

Kroupa, P. 2005, The fundamental building blocks of galaxies, in ESA SP-576: The ThreeDimensional Universe with Gaia, 629 (astro-ph/0412069)

Kroupa, P. \& Boily, C. M. 2002, MNRAS, 336, 1188

Kroupa, P. \& Weidner, C. 2003, ApJ, 598, 1076

Kroupa, P. \& Weidner, C. 2005, in Massive Star Birth: A Crossroads of Astrophysics, IAUS 227, 423

Kroupa, P., Aarseth S.J., Hurley, J. 2001, MNRAS, 321, 699

Krumholz, M. R. \& Tan, J. C. 2007 ApJ, 654, 304

Lada, C. J. \& Lada, E. A. 2003, ARA\&A, 41, 57

Li, Y., Klessen, R. S., \& Mac Low, M.-M. 2003, ApJ, 592, 975

Mac Low, M.-M. \& Klessen, R. S. 2004, Reviews of Modern Physics, 76, 125

Martins, F., Schaerer, D., \& Hillier, D. J. 2005, A\& $A$, 436, 1049

McMillan, S. L. W., Vesperini, E., \& Portegies Zwart, S. F. 2007, ApJ655, L45

Odenkirchen, M., et al. 2003, AJ, 126, 2385

Oey, M. S. \& Clarke, C. J. 2005, ApJ, 620, L43

Palla, F. \& Stahler, S. W. 2000, ApJ, 540, 255

Palla, F., Randich, S., Pavlenko, Y. V., Flaccomio, E., \& Pallavicini, R. 2007, ApJ, 659, L41

Parker, R. J. \& Goodwin, S. P. 2007, MNRAS, 380, 1271

Parmentier, G., Gilmore, G. 2005, MNRAS, 363, 326 (2005)

Parmentier, G. \& Gilmore, G. 2007, MNRAS, 377, 352

Parmentier, G., Goodwinj, S., Kroupa, P., \& Baumgardt, H. 2008, ApJ, submitted

Pflamm-Altenburg, J. \& Kroupa, P. 2006, MNRAS, 373, 295

Pflamm-Altenburg, J. \& Kroupa, P. 2007, MNRAS, 375, 855

Portegies Zwart, S. F., McMillan, S. L. W., Hut, P., \& Makino, J. 2001, MNRAS, 321, 199

Scally, A., \& Clarke, C. 2002, MNRAS, 334, 156

Stamatellos, D., Whitworth, A. P., Bisbas, T., \& Goodwin, S. 2007, A\&AA, 475, 37

Testi, L., Sargent, A. I., Olmi, L., \& Onello, J. S. 2000, ApJ, 540, L53

Tilley, D. A. \& Pudritz, R. E. 2007, MNRAS, 930

Vesperini, E. 1998, MNRAS, 299, 1019

Vesperini, E. 2001, MNRAS, 322, 247

Weidner, C. \& Kroupa, P. 2004, MNRAS, 348, 187

Weidner, C. \& Kroupa, P. 2005, ApJ, 625, 754

Weidner, C. \& Kroupa, P. 2006, MNRAS, 365, 1333

Weidner, C., Kroupa, P., \& Larsen, S. S. 2004, MNRAS, 350, 1503

Weidner, C., Kroupa, P., Nürnberger, D. E. A., \& Sterzik, M. F. 2007, MNRAS, 376, 1879

Wuchterl, G. \& Tscharnuter, W. M. 2003, A\&A, 398, 1081

Zinnecker, H. \& Yorke, H. W. 2007, ARAEA A, 45, 481 